\title{
Airways dilate to simulated inspiratory but not expiratory manoeuvres
}

\author{
Adrian R. West*,\#, Elangovan Thaya Needi*, Howard W. Mitchell*, \\ Peter K. McFawn* and Peter B. Noble*, ${ }^{\star,+}$
}

ABSTRACT: In a healthy human, deep inspirations produce bronchodilation of contracted airways, which probably occurs due to the transient distension of the airway smooth muscle (ASM). We hypothesised that deep expiratory manoeuvres also produce bronchodilation due to transient airway wall and ASM compression.

We used porcine bronchial segments to assess the effects of deep inspirations, and maximal and partial expiration (submaximal) on airway calibre. Respiratory manoeuvres were simulated by varying transmural pressure using a hydrostatic pressure column: deep inspiration 5 to $30 \mathrm{cmH}_{2} \mathrm{O}$, maximal expiration 30 to $-15 \mathrm{cmH}_{2} \mathrm{O}$, partial expiration 10 to $-15 \mathrm{cmH}_{2} \mathrm{O}$; amidst a background of tidal oscillations, 5 to $10 \mathrm{cmH}_{2} \mathrm{O}$ at $0.25 \mathrm{~Hz}$. Changes in luminal cross-sectional area in carbachol-contracted airways were measured using video endoscopy.

Deep inspirations produce an immediate bronchodilation $(\sim 40-60 \%, p=0.0076)$ that lasts for up to $1 \mathrm{~min}(p=0.0479)$. In comparison, after maximal expiration there was no immediate change in airway calibre; however, a delayed bronchodilatory response was observed from $4 \mathrm{~s}$ after the manoeuvre $(p=0.0059)$ and persisted for up to $3 \mathrm{~min}(p=0.0182)$. Partial expiration had little or no effect or airway calibre.

The results observed demonstrate that the airway wall dilates to deep inspiration manoeuvres but is unresponsive to deep expiratory manoeuvres.

KEYWORDS: Airway calibre, asthma, bronchodilation, deep inspiration, maximal expiration, spirometry

$\mathrm{n}$ healthy humans, breathing manoeuvres such as deep inspirations can dilate previously contracted airways [1-6] and represent a potent physiological mechanism for maintaining airway calibre. The importance of this regulatory pathway is demonstrated by observations that deep inspiration responses are reduced or absent in obstructive diseases, such as asthma $[1,2,5]$ and chronic obstructive pulmonary disease (COPD) $[7,8]$. The bronchodilator actions of deep inspiration in vivo could be explained by an intrinsic response of the airway wall to stretch due to the transmural pressure gradients that are generated during lung inflation. In vitro, dynamic inflation produces bronchodilation of contracted airway segments $[9,10]$. The magnitude and duration of bronchodilation in airway segments is comparable with that observed in vivo suggesting that the actions of deep inspirations are initiated at airway level $[3,10]$.

The clinical impact of bronchodilation after a deep inspiration is revealed in tests, such as forced expiratory volume in $1 \mathrm{~s}$ (FEV1) or forced vital capacity (FVC). Critically, these manoeuvres are preceded by deep inspirations that produce bronchodilation, which itself may influence the clinically and experimentally derived lung function data. For instance, when assessing patients with an obstructive disease the absence or presence of a deep inspiration determines the extent of airway hyperresponsiveness observed in this group [1, 11]. Due to these complicating effects respiratory manoeuvres that do not involve deep inspirations have been used as an alternative, most notably partial forced expiration $[2,12-14]$. Although used with the intention of avoiding the deep inspiration phase of an FEV1, it is unknown whether expiratory phases of the FEV1, or other expiratory manoeuvres, can themselves influence airway calibre.

While a deep inspiration and a deep expiration initially exert opposing effects on airway calibre (stretch versus compression), evidence from in vitro studies suggest that it is the dynamic nature of the movements that ultimately determines the airway response. In particular, change in length or length oscillation of airway smooth muscle (ASM) in vitro reduces muscle force $[15,16]$.

\section{AFFILIATIONS}

*Physiology, School of Biomedical, Biomolecular and Chemical Sciences, The University of Western Australia,

'School of Women's and Infants' Health, The University of Western Australia, and

${ }^{+}$Centre for Neonatal Research and Education, The University of Western Australia, Perth, Australia.

${ }^{\text {\#}}$ School of Biomedical Engineering, Dalhousie University, Halifax, NS, Canada.

CORRESPONDENCE

P.B. Noble

M094, School of Women's and Infants' Health

The University of Western Australia 35 Stirling Hwy

Crawley

6009

Australia

E-mail: Peter.Noble@uwa.edu.au

Received:

Oct 272011

Accepted after revision: Dec 132011

First published online:

Jan 262012 
However, adaptive properties (plasticity) of ASM are equally sensitive to both increases and decreases in length [17] and this represents a plausible mechanism underlying the response of the ASM to dynamic stretch associated with breathing manoeuvres [18]. Thus, both mechanical stretch and compression may contribute to the effects on airway calibre elicited by breathing manoeuvres. Importantly, if expiration does exert similar effects to inspiration, then the expiratory phases of FEV1, FVC and indeed partial expiration will also favour bronchodilation and influence these measures of airway calibre or responsiveness.

The aim of the present study was to characterise the response of the airway wall separately to both inspiratory and expiratory manoeuvres, including deep inspiration (inflation to total lung capacity (TLC)), maximal expiration (inflation to TLC then deflation to residual volume (RV)), and partial expiration (deflation from end tidal volume to RV). We were particularly interested in whether expiratory phases of clinically relevant parameters, such as FEV1, FVC or partial expiration, could regulate airway calibre by producing bronchodilation. Based on our previous findings $[9,10]$ we hypothesised that bronchodilatory responses to the different breathing manoeuvres would be expressed at the level of the airway without the need for contributions from airway-parenchymal mechanical interactions or central neural reflexes. Bronchial segments from pigs were contracted to carbachol and bronchodilator responses to each breathing manoeuvre were recorded by video endoscopy. Respiratory manoeuvres were simulated by varying the transmural pressure of the airway, which were applied by a dynamically oscillating pressure column.

\section{METHODS}

\section{Animal handling}

All animal experiments conformed to the institutional ethics and animal care unit regulations (Animal Ethics Committee, University of Western Australia, Perth, Australia). White Landrace pigs $(\sim 30 \mathrm{~kg})$ were initially sedated with tiletamine/zolazepam (4.4 mg. $\mathrm{kg}^{-1}$ i.m.) (Virbac, Peakhurst, Australia) and xylazine (2.2 mg. $\mathrm{kg}^{-1}$ i.m.) (Troy Laboratories, Smithfield, Australia) and then exsanguinated under pentobarbitone sodium anaesthesia (30 mg $\cdot \mathrm{kg}^{-1}$ i.v.). The lungs were then removed and transported, on ice, to the laboratory for dissection of the airways.

\section{Airway preparation}

A length of the bronchial tree was dissected from the lower lobe of the right lung, beginning from a lobar bronchus and extending distally by $\sim 5 \mathrm{~cm}$. All side branches were ligated producing a leak-free preparation. The two ends of the bronchus were cannulated and the tissue preparation was placed horizontally in a custom made perspex organ bath containing gassed $\left(95 \% \mathrm{O}_{2}\right.$ and $\left.5 \% \mathrm{CO}_{2}\right)$ Krebs solution $(\mathrm{NaCl} 121 \mathrm{mM} ; \mathrm{KCl}$ $5.4 \mathrm{mM} ; \mathrm{MgSO}_{4} 1.2 \mathrm{mM} ; \mathrm{NaHCO}_{3} 25 \mathrm{mM}$; sodium morpholinopropane sulfonic acid $5.0 \mathrm{mM}$; glucose $11.5 \mathrm{mM}$; and $\mathrm{CaCl}_{2}$ $2.5 \mathrm{mM}$ ) at $37^{\circ} \mathrm{C}$. The airway segment was stretched to a length shown previously to approximate functional residual capacity in the pig lung, i.e. $\sim 105 \%$ of the fully deflated length at $0 \mathrm{cmH}_{2} \mathrm{O}$ [19].

\section{Video endoscopy}

Changes in airway lumen calibre (i.e. cross sectional area) to bronchoconstrictor agonist and breathing manoeuvres were recorded by video endoscopy, as previously described [20]. Unlike morphometry, measurements obtained by this approach do not include the area or perimeter contained between interstices. A rigid fibreoptic endoscope (Olympus SES-1711D; Olympus, Center Valley, PA, USA) coupled to a video camera (DFW-SX900; Sony, Tokyo, Japan) was inserted into the lumen of the airway through the proximal cannula. The endoscope was locked at a position suitable to visualise airways of generation between 10 and 12 (trachea=generation 0 ). Prior to recordings the airway lumen was stained with a blue ring of dye using a steel applicator to aid visualisation. Colour video images of the lumen were displayed in real time and recorded on a personal computer using video-acquisition software (Fire-I 1.21; Unibrain, San Ramon, CA, USA).

\section{Simulation of respiratory manoeuvres}

Respiratory manoeuvres were simulated by controlling airway transmural pressure, which was positive for tidal breathing and deep inspiration, and negative for deep expiration. Transmural pressure was set by the height of a hydrostatic pressure column connected in series with the airway lumen. By using this approach the airway luminal pressure, and therefore transmural pressure, was determined by the relative height of the pressure column to the midline of the segment. Hence transmural pressure was positive when the height of the column exceeded the airway midline and negative when the height of the column was below the airway midline. Dynamic breathing manoeuvres (as discussed later) were simulated by cycling the height of the pressure column using a computercontrolled syringe pump. The syringe plunger was driven by a direct current motor (M540; McLennan Servo Supplies, Ash Vale, UK) using a BioPWM sequential motor controller (V0.3) and custom designed software (provided by S. De Catania; University of Western Australia, Perth, Australia), which allowed for sinusoidal or ramp movements at a desired frequency. The syringe pump was calibrated to establish the linear relationship between syringe displacement and the change in fluid height in the pressure column. Pressure changes were confirmed by a transducer (MPX2010DP; Motorola Inc., Schaumburg, IL, USA) connected to an airway lumen port in the organ bath. Pressure signals were recorded using a PowerLab 2/20 data-acquisition system (ADInstruments Pty Ltd, Bella Vista, Australia).

\section{Experimental protocol}

Airway preparations were allowed $1 \mathrm{~h}$ to equilibrate with the organ bath conditions before experimentation commenced, during which time the lumen and adventitia of the segment were regularly flushed with fresh Krebs solution. Tissue viability was confirmed by observing airway contractions to acetylcholine $(0.001 \mathrm{M})$ followed by a 30 -min washout and recovery period.

The volume history and dynamic environment of the airway was initially set by three deep inspirations followed by $20 \mathrm{~min}$ of tidal oscillations, simulated by sinusoidal transmural pressure cycles between 5 and $10 \mathrm{cmH}_{2} \mathrm{O}$ at $0.25 \mathrm{~Hz}$ (i.e. the human breathing frequency). Tidal oscillations were continued and airways were contracted to $10^{-6} \mathrm{M}$ carbachol administered to the adventitial surface of the airway, a median effective concentration (EC50) concentration that produced $\sim 35 \%$ decrease in lumen area. Preliminary studies showed that airway narrowing 
was stable $30 \mathrm{~min}$ after the addition of carbachol, and remained so for at least a further $10 \mathrm{~min}$ (see results section). Therefore, our approach was to induce a breathing manoeuvre $30 \mathrm{~min}$ after the addition of carbachol and then track the airway lumen area for 10 min post-manoeuvre. At the end of the recording period the carbachol was replaced with fresh Krebs solution and the airway was allowed to relax for $40 \mathrm{~min}$, with regular flushing of both adventitial and luminal surfaces. The entire protocol was then repeated for the next breathing manoeuvre.

\section{Dynamic breathing manoeuvres}

Three breathing manoeuvres were administered to each airway in a random order. A deep inspiration comprised of a linear ramp increase in pressure from 5 to $30 \mathrm{cmH}_{2} \mathrm{O}$, a $2 \mathrm{~s}$ pause at end inspiration, and a ramp decrease in pressure to $5 \mathrm{cmH}_{2} \mathrm{O}$. Partial expiration comprised of a ramp decrease in pressure from 5 to $-15 \mathrm{cmH}_{2} \mathrm{O}$, a $2 \mathrm{~s}$ pause at end expiration, and a ramp increase in pressure to $5 \mathrm{cmH}_{2} \mathrm{O}$. Maximal expiration effectively combined the two previous manoeuvres, replicating the initial phase of deep inspiration including the $2 \mathrm{~s}$ pause at $30 \mathrm{cmH}_{2} \mathrm{O}$ (end inspiration), followed by a large ramp decrease in pressure to $-15 \mathrm{cmH}_{2} \mathrm{O}$, a $2 \mathrm{~s}$ pause (end expiration), and finally a ramp increase in pressure to $5 \mathrm{cmH}_{2} \mathrm{O}$. Respective end inspiratory and expiratory pressures of $30 \mathrm{cmH}_{2} \mathrm{O}$ and $-15 \mathrm{cmH}_{2} \mathrm{O}$ meant that pressure amplitudes were comparable between inspiratory and expiratory manoeuvres (i.e. from a mean pressure of $7.5 \mathrm{cmH}_{2} \mathrm{O}$ during tidal oscillation, inspiration to $30 \mathrm{cmH}_{2} \mathrm{O}$ or expiration to $-15 \mathrm{cmH}_{2} \mathrm{O}$, change in pressure was $22.5 \mathrm{cmH}_{2} \mathrm{O}$ ). The rate of pressure change was kept constant for all manoeuvres and was $12.5 \mathrm{cmH} \mathrm{H}_{2} \mathrm{O} \cdot \mathrm{s}^{-1}$. Example pressure traces indicating tidal oscillations, all three breathing manoeuvres, and associated measurement points are shown in figure 1.

\section{Analysis and statistics}

Lumen cross sectional area was quantified by manually tracing an area around the bronchial lumen using ImageJ 1.44 (National Institutes of Health, Bethesda, MD, USA). Images were calibrated using a probe of known diameter inserted into the lumen. To assess the potential bronchodilatory effects of each manoeuvre, airway lumen area $(A)$ at different postmanoeuvre time-points $(t)$ were expressed as the percentage recovery in airway narrowing to carbachol as follows:

$$
\text { Recovery } \%=\frac{A(t)-A_{\text {pre-manoevre }}}{A_{\text {pre-carbachol }}-A_{\text {pre-manoeuvre }}}
$$

where Apre-manoeuvre is lumen area prior to the initiation of the simulated breathing manoeuvre (contracted to carbachol) and Apre-carbachol is lumen area of the relaxed airway prior to carbachol. A positive percentage recovery indicated bronchodilation (i.e. $100 \%$ recovery corresponds to complete reversal of constriction) while a negative percentage recovery indicated additional contraction. To quantify luminal strain (linear) during each respiratory manoeuvre changes in lumen perimeter $(P)$ at end inspiration and expiration were calculated as follows:

$$
\text { Lumen strain } \%=\frac{p_{\text {insp } / \text { exp ir }}-p_{\text {pre-manouevre }}}{p_{\text {pre-manouevre }}}
$$

where Pinspir/expir is lumen perimeter at end inspiration or expiration and Ppre-manoeuvre is lumen perimeter prior to manoeuvre (contracted to carbachol). A positive lumen strain indicated airway expansion and negative strain compression. Airway shape at baseline, after contraction to carbachol and during the inspiratory and expiratory manoeuvres, was assessed using the circularity index. The circularity index was calculated from the ratio of the measured lumen area to that predicted from perimeter assuming circularity (i.e. $4 \pi \times$ area/ perimeter $^{2}$ where a ratio of one indicates a perfect circle).

All data are expressed as mean \pm SEM, and all statistics were performed with GraphPad Prism (v4.03; GraphPad Software, San Diego, CA, USA) and Statistica (StatSoft Inc., Tulsa, OK, USA). Repeat measures one-way ANOVA was used to compare relaxed airway lumen area (i.e. airway size) and the magnitude of airway narrowing prior to the initiation of the

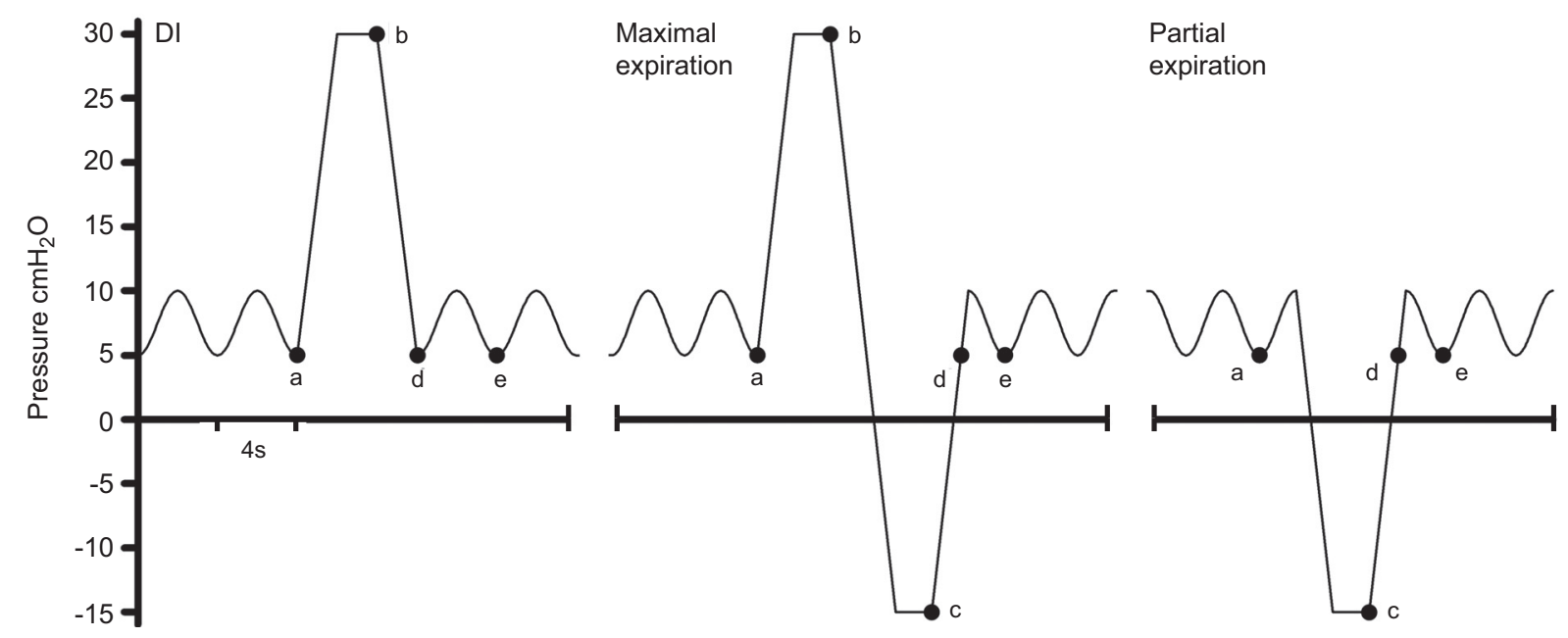

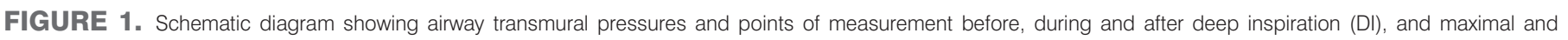

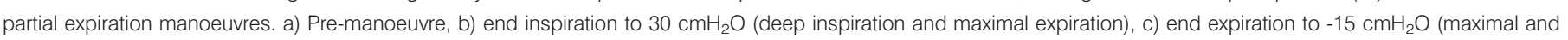

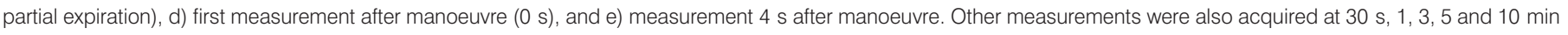
after manoeuvres, and prior to the administration of carbachol (i.e. relaxed airway). 


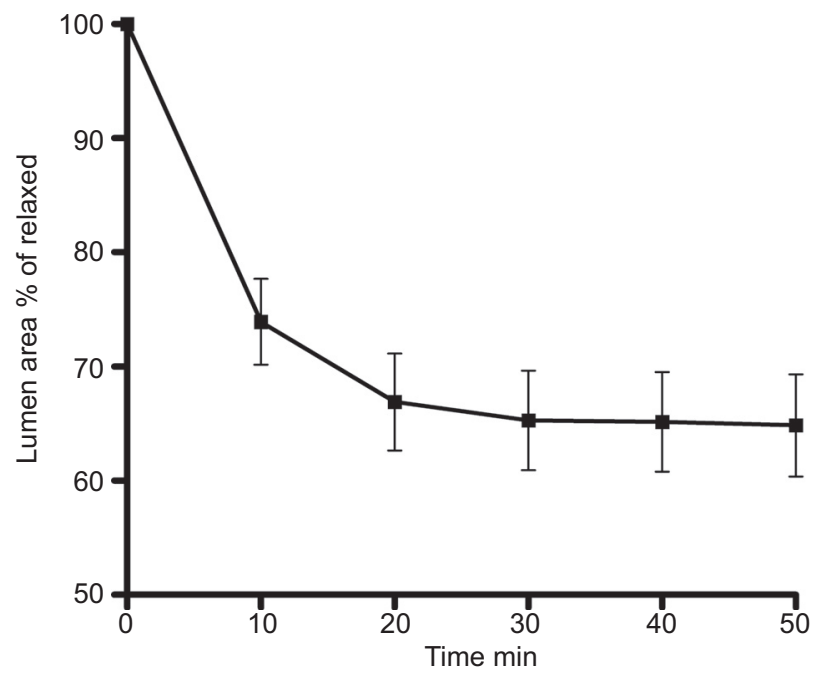

FIGURE 2. Airway narrowing time course study to carbachol $(n=4)$. Lumen area, measured as percentage of relaxed airway, was measured during tidal oscillations alone. Lumen area plateaued $30 \mathrm{~min}$ after contraction and remained stable thereafter.

manoeuvres. The presence of bronchodilation or bronchoconstriction following a manoeuvre was assessed by two-tailed one sample t-test against a hypothesised mean of zero (no change in luminal area). Comparisons between bronchodilation following deep inspiration and maximal expiration was assessed by two-way ANOVA and Newman-Keuls post hoc tests, with manoeuvre and time as repeat variable measures. Lumen perimeter strains at end inspiration (deep inspiration versus maximal expiration) and end expiration (maximal expiration versus partial expiration) were compared using a paired t-test, while repeat measures one-way ANOVA was used to compare strains measured at end inspiration versus end expiration (of note, for this analysis absolute strain was compared; see results section). For consistency, measured lumen perimeter strains were also assessed by two-tailed one sample t-test against a hypothesised mean of zero. A $p<0.05$ was considered statistically significant.

\section{RESULTS}

\section{Airway narrowing to carbachol}

In a separate group of airways $(n=4)$ the airway narrowing time course to a submaximal dose of carbachol was assessed (fig. 2). In the presence of tidal oscillation, carbachol produced an $\sim 35 \%$ reduction in lumen area that reached a plateau at
$30 \mathrm{~min}$ and remained stable for up to $50 \mathrm{~min}$ after the addition of carbachol. Based on these observations we chose to assess the response to each manoeuvre at $30 \mathrm{~min}$ after the addition of carbachol, and to monitor the resulting changes in lumen area for $10 \mathrm{~min}$. The magnitude of airway narrowing prior to each breathing manoeuvre was the same (table 1).

\section{Effect of inspiration and expiration on airway narrowing}

We assessed the effects of both inspiratory (deep inspiration) and expiratory (maximal and partial expiration) manoeuvres on airway narrowing. A sample image sequence for maximal expiration that comprises airway narrowing to carbachol, an initial deep inspiration (airway expansion) and a subsequent expiratory manoeuvre (airway compression) is shown in figure 3. In the example, airway expansion at end inspiration (increased lumen area) and compression (reduced lumen area) at end expiration are evident.

To examine the potential bronchodilatory effects of each respiratory manoeuvre, changes in airway lumen area after each manoeuvre (deep inspiration, and maximal and partial expiration) were expressed as the percentage recovery in airway narrowing (fig. 4). Consistent with our previous findings [9], deep inspiration produced immediate bronchodilation $(\mathrm{p}=$ $0.0076)$ that persisted for up to $1 \mathrm{~min}$ after the manoeuvre $(p=0.0479)$, subsiding within $3 \mathrm{~min}(\mathrm{p}=0.1803)$. In comparison, immediately after maximal expiration $(0 \mathrm{~s})$ the airway was neither contracted nor dilated, but exhibited significant dilation from $4 \mathrm{~s}(p=0.0059)$ to $3 \mathrm{~min}(p=0.0182)$ but not at $5 \mathrm{~min}$ $(p=0.0917)$. The magnitude of bronchodilation to maximal expiration at $4 \mathrm{~s}$ was $15.0 \pm 1.2 \%$ recovery which was less than the $28.7 \pm 2.2 \%$ recovery after deep inspiration at the same timepoint $(\mathrm{p}=0.0002)$. At all other subsequent time-points there was no significant difference in percentage of recovery between deep inspiration and maximal expiration. In stark contrast to both deep inspiration and maximal expiration, partial expiration failed to produce any dilation, with some possible initial further constriction (NS, $\mathrm{p}=0.1367$ ) that was completely absent by $4 \mathrm{~s}$.

\section{Luminal strain during inspiration and expiration}

To quantify the extent of airway wall expansion and/or compression during each respiratory manoeuvre, luminal perimeter strain (percentage) was calculated at end inspiration and end expiration (fig. 5) and these were all statistically significant from zero as assessed by one sample t-tests. Luminal strain was $18.6 \pm 6.6 \%$ and $15.1 \pm 3.1 \%$ at end inspiration for deep inspiration and maximal expiration, respectively, and $-21.2 \pm 3.4 \%$ and $-23.9 \pm 3.0 \%$ at end expiration for maximal and partial expiration, respectively. There was no statistical

\section{TABLE 1 Airway lumen area before and after narrowing to carbachol}

\begin{tabular}{lcccc} 
Manoeuvre & Subjects $\mathbf{~}$ & Relaxed $\mathbf{~ m m}^{\mathbf{2}}$ & Contracted $\mathbf{m m}^{\mathbf{2}}$ & Airway narrowing \% \\
\hline Deep inspiration & 5 & $14.2 \pm 2.7$ & $9.2 \pm 2.6$ & $38.6 \pm 8.1$ \\
Partial expiration & 5 & $14.8 \pm 2.6$ & $8.7 \pm 1.9$ & $35.3 \pm 6.4$ \\
Maximal expiration & 5 & $13.4 \pm 2.9$ & $9.5 \pm 1.9$ & $35.1 \pm 4.9$ \\
\hline
\end{tabular}

Data are presented as mean \pm SEM, unless otherwise stated. Airway narrowing was calculated from the percentage reduction in lumen area. Prior to each manoeuvre lumen area $(p=0.6166)$ and airway narrowing $(p=0.8005)$ were similar between groups. 

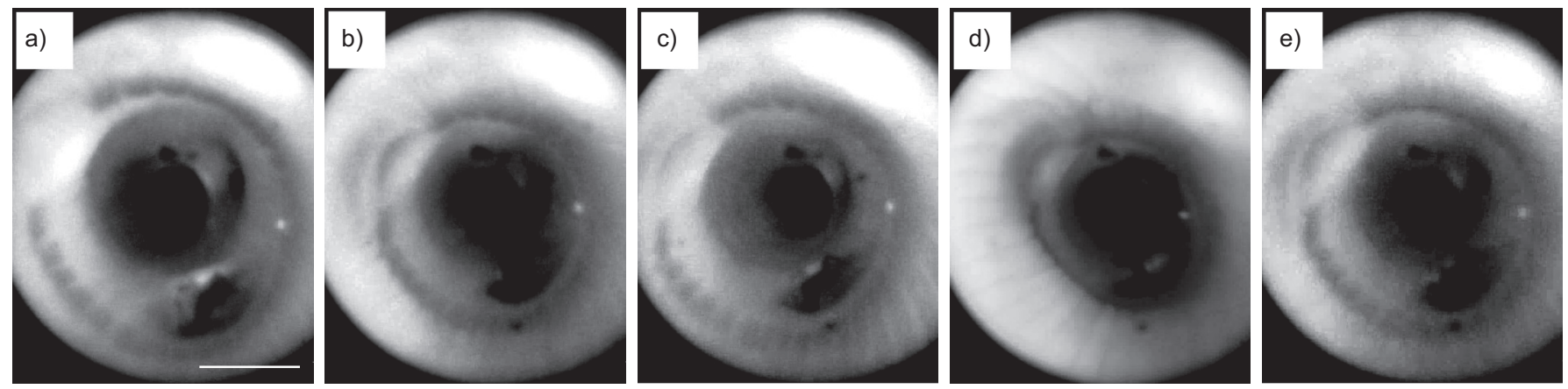

FIGURE 3. Sample image sequence for a maximal expiration manoeuvre. Lumen images were taken prior to a) and after b) narrowing to carbachol, at end inspiration c) and end expiration $\mathrm{d}$ ) and at several time-points after the manoeuvre (e, $4 \mathrm{~s}$ ). Typically there was no change in airway lumen immediately after maximal expiration, but dilation was apparent at $4 \mathrm{~s}$. Scale bar $=2 \mathrm{~mm}$.

difference in the magnitude of strain at end inspiration between deep inspiration and maximal expiration $(\mathrm{p}=0.4599)$ or at end expiration between maximal and partial expiration $(\mathrm{p}=0.4376)$. When direction of strain was ignored (i.e. expansion versus compression) there was no difference between the magnitude of strain (absolute strain) at end inspiration compared with end expiration $(\mathrm{p}=0.5753)$.

Finally, we considered the possibility that if the lumen became less circular, particularly during the compressive manoeuvre, this may limit the amount of ASM strain. However, we saw no gross changes in lumen dimensions and this was confirmed by the circularity index ( $>0.94$ under all conditions).

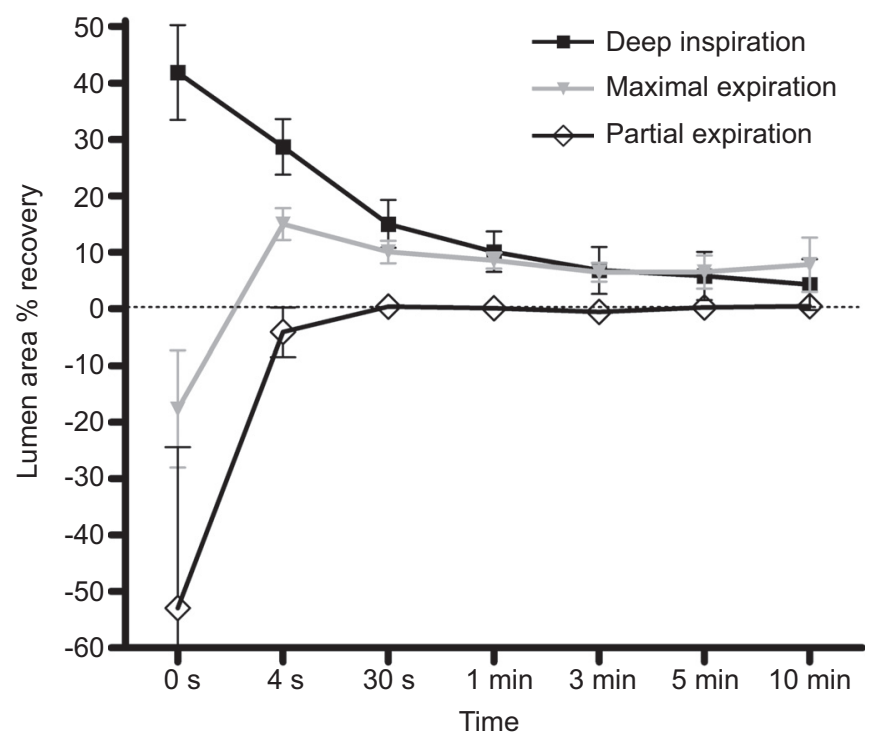

FIGURE 4. Percentage recovery $(n=5)$ in airway narrowing after deep inspiration, and maximal and partial expiration. Deep inspiration produced immediate bronchodilation $(p=0.0076)$ that persisted for at least $60 \mathrm{~s}$. The airway was neither dilated nor contracted immediately after maximal expiration; however a delayed bronchodilatory response was observed from $4 \mathrm{~s}(p=0.0059)$ to $180 \mathrm{~s}$ after manoeuvre $(p=0.0182$ ). In contrast, partial expiration did not produce a statistically significant change in airway calibre (and importantly no bronchodilation), although there was a trend towards enhanced narrowing immediately after the manoeuvre (not significant $p=0.1367$ ), but which was absent $4 \mathrm{~s}$ later.

\section{DISCUSSION}

The present study determined whether airway calibre, which is known to be regulated by deep inspiratory manoeuvres, is also modulated by deep expiratory manoeuvres. Building on previous findings that airway responses to respiratory movements are initiated by direct stretch on the airway wall $[9,10,21,22]$, we used an isolated airway model in vitro and simulated respiratory manoeuvres by varying transmural pressure. Our findings show that airways exhibit an intrinsic bronchodilatory response to high positive transmural pressures accompanying lung inflation, but not to negative (compressive) transmural pressures achieved during deep expiration.

We evaluated the effects of three different respiratory manoeuvres: 1) deep inspiration, involving large positive transmural pressures $\left(30 \mathrm{cmH}_{2} \mathrm{O}\right)$ that distended (stretched) the airway wall; 2) partial expiration, which compressed the airway wall to negative transmural pressures $\left(-15 \mathrm{cmH}_{2} \mathrm{O}\right)$; and 3) maximal expiration, which essentially combined the expansive and compressive manoeuvres (i.e. 30 to $-15 \mathrm{cmH}_{2} \mathrm{O}$ ). Results show that the airway wall exhibits an intrinsic bronchodilatory response to

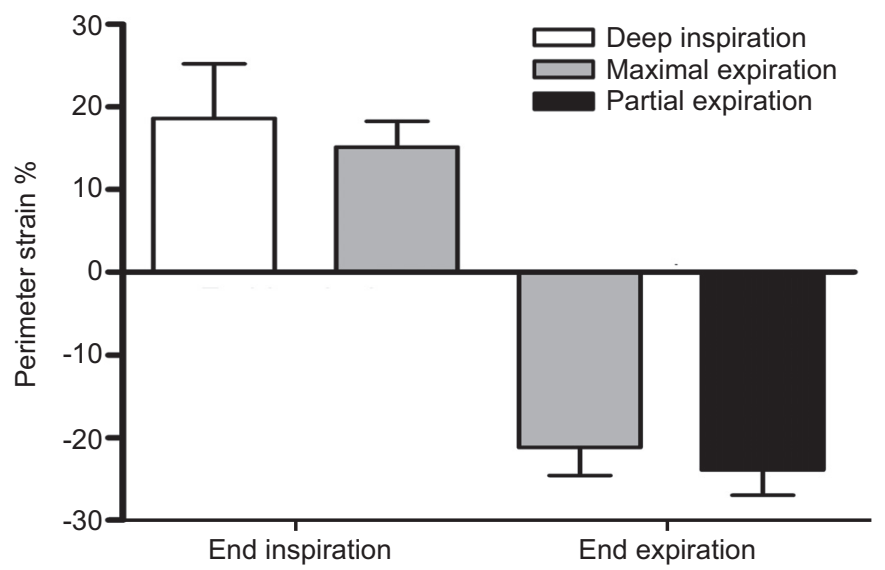

FIGURE 5. Lumen perimeter strain $(n=5)$ measured at end inspiration, during deep inspiration and maximal expiration, and at end expiration, during maximal and partial expiration. There was no statistical difference in the magnitude of strain at end inspiration between deep inspiration and maximal expiration $(p=0.4599)$ or at end expiration between maximal expiration and partial expiration $(p=0.4376)$. There was also no difference between the magnitude of strain (absolute strain) at end inspiration compared with end expiration $(p=0.5753)$. 
mechanical stretch accompanying a deep inspiration, confirming our earlier findings $[9,10,22]$; however there is no such bronchodilatory response to airway compression accompanying deep expiration (partial expiration). In contrast, the airway tends to be more constricted immediately after a partial expiration, although this was not statistically significant $(p=0.1367)$ and any meaningful change is completely absent $4 \mathrm{~s}$ later. Finally, with respect to maximal expiration, which involves an initial deep inspiration, the dominant response was bronchodilation, albeit delayed, as the magnitude of bronchodilation was partially offset by wall compression achieved during the subsequent deep expiratory manoeuvre.

Our methodological approach closely followed our previous study where positive transmural pressures simulating deep inspiration produced bronchodilation in contracted bronchial segments in vitro [9]. The precise transmural pressures that occur during the different breathing manoeuvres in vivo are difficult to predict, but we defined deep inspiration as inflation to $30 \mathrm{cmH}_{2} \mathrm{O}$ since this corresponds to the plateau in the pressure-volume curve of the airway [23]. With respect to maximal and partial expiration, we simulated airway wall compression at negative (subatmospheric) transmural pressures present during deep expiratory movements, which may also involve dynamic airway compression. End expiration was arbitrarily defined as $-15 \mathrm{cmH}_{2} \mathrm{O}$ so that inflationary and deflationary manoeuvres produced a similar amplitude change (i.e. change in pressure was $22.5 \mathrm{cmH}_{2} \mathrm{O}$ ). We also chose to use a submaximal, but physiologically relevant, level of airway narrowing. Given that the level of airway contraction (narrowing) impacts considerably on the response to respiratory manoeuvres [22], airways were narrowed to an approximate EC50 dose. Carbachol produced an $\sim 35 \%$ reduction in lumen area, which in vivo would have a meaningful effect on airflow (a $60 \%$ reduction, assuming homogenous constriction and laminar flow) while still not maximally contracting ASM.

During standard lung function testing (i.e. $\mathrm{FEV}_{1}$ and $\mathrm{FVC}$ ) a deep inspiration that precedes maximal expiration produces bronchodilation and, therefore, influences subsequent expiratory flow $[2,24]$. The potential impact of bronchodilation to deep inspiration during the assessment of airway responsiveness/hyperresponsiveness has been documented, although the underlying implications are still not fully appreciated. Since the magnitude of the bronchodilatory response to deep inspiration differs in disease including asthma and COPD [1, 2, 5, 7, 8], the presence or absence of deep inspiration during bronchial challenges will change the severity of the hyperresponsiveness observed. Indeed if deep inspirations are removed from bronchial challenges dose-response curves from healthy and asthmatic individuals converge [1, 11]. Any bronchodilatory response to expiration could further complicate this scenario; however the present data now supports the assumption that expiratory manoeuvres do not influence airway calibre by regulating ASM force.

As discussed earlier, there was also a tendency for airways to be more constricted immediately after the expiratory phase of a manoeuvre that was of borderline significance and lasting only 4 s. Residual airway compression after expiration is likely to reflect the inertial and viscous properties of the airway wall (i.e. the airway will not return to its precompressed lumen area instantly when the compressive load is removed) and thus a transient mechanical effect rather than a biological response to an applied mechanical stimulus. How these mechanical effects would impact lung function testing is unclear, since in this clinical scenario the flow profiles are assessed during the expiratory manoeuvre rather than after re-inflation, as was the case in our protocol. Irrespective of the above considerations, the major conclusion of the present study remains firm: the airway wall does not respond actively to compressive manoeuvres by producing bronchodilation analogous to deep inspiration.

There is compelling evidence that bronchodilation to deep inspiration observed in vivo [1-6] is mediated by direct stretch to the airway wall that lengthens ASM and produces a reduction in force generation $[9,10,15,16,21,22]$. Bronchodilation to radial stretch simulating deep inspiration is the prevailing response in human and porcine bronchial segments [9, 10], although under some conditions modest contractile responses have been observed in pigs [25], which may bare some relationship to bronchoconstrictor responses to deep inspiration observed in asthmatic individuals [26]. Reduced ASM force after mechanical stretch may be due to cross-bridge detachment [15] and/or arise as a result of adaptive ASM properties involving the reorganisation of contractile filaments [18]. However, while it is ASM lengthening or mechanical stretch that is more often linked to the ASM response, it may be length change (lengthening or shortening) that ultimately matters and the underlying cellular mechanisms may also be responsive to muscle shortening/compression. Indeed, adaptive properties of ASM are equally responsive to both lengthening and shortening, whereby a chronic increase or decrease in ASM length alters length-tension characteristics [17]. It is also feasible that cross-bridge binding may be sensitive to cellular compression, although to our knowledge this has not been examined previously. In the present study we hypothesised that expiratory manoeuvres, involving ASM compression, could also favour a reduction in ASM force and bronchodilation. Our data does not support this possibility.

One possible explanation for the lack of an effect of expiration on airway calibre is that the amplitude of ASM length change during the manoeuvre falls below that required to initiate a reduction in the ASM force. Several studies have shown that the magnitude of bronchodilation depends on the amplitude of the applied stretch or pressure change [4, 9, 15, 21, 22]. Although we applied the same change in pressure during both inflation and deflation, it is possible that the strain applied to the ASM may have been less in the expiratory manoeuvres, for example if the airway wall was more resistant to compression than expansion. While it is very difficult to assess dynamic ASM length change in situ, in the present study we used airway luminal perimeter strain measured during inspiratory (positive strain) and expiratory (negative strain) phases of the manoeuvres. Results suggest that inspiratory and expiratory manoeuvres produced similar airway wall strain and given that bronchodilation was observed in response to inspiration, the level of airway strain was sufficient to modify the ASM force.

However, it is important to consider that the lumen perimeter strain is not a direct proxy for ASM strain. The airway wall, internal to the muscle, may become thicker during a compressive manoeuvre and comparatively thinner during expansion. 
Luminal perimeter strain may then overestimate ASM strain during expiration. Data from a previous study, which used anatomical optical coherence tomography to measure wall and luminal dimensions [27], was used to account for any disparity between ASM and luminal perimeter due to the thickness of the inner wall. These data were used to predict changes in ASM perimeter from the measured changes in luminal area assuming circularity and constant wall area. The results of the analysis indicated comparable ASM strain during inspiration and expiration (15\% and $-18 \%$ strain, respectively). Studies on isolated ASM [15] suggest that length changes exceeding $4 \%$ will produce large reductions in ASM force, therefore, it seems unlikely that a reduced response to deep expiration (i.e. lack of bronchodilation) can be explained by an applied ASM strain below that sufficient to regulate an ASM force. A final possibility for the failure of expiration to produce bronchodilation is that the airway folds on compression without a change in ASM length, although in the present study we saw no evidence for gross changes in lumen shape during deflation (fig. 3) and this was confirmed by a lack of change in the circularity index.

In conclusion, we have found strong support for the view that deep expiratory manoeuvres do not cause bronchodilation, supporting the notion that partial expiratory manoeuvres can be used to assess bronchoconstriction without contaminating the measurement by themselves changing the ASM tone. These findings suggest that while the ASM is sensitive to dynamic mechanical stretch, it is unresponsive to transient compressive events.

\section{SUPPORT STATEMENT}

The study was supported by project funding from the NHMRC of Australia (No. 513842) and the Raine Medical Research Foundation. P.B. Noble's salary was provided by an NHMRC Biomedical Fellowship (No. 513921)

\section{STATEMENT OF INTEREST}

None declared.

\section{REFERENCES}

1 Brusasco V, Crimi E, Barisione G, et al. Airway responsiveness to methacholine: effects of deep inhalations and airway inflammation. J Appl Physiol 1999; 87: 567-573.

2 Crimi E, Pellegrino R, Milanese M, et al. Deep breaths, methacholine, and airway narrowing in healthy and mild asthmatic subjects. J Appl Physiol 2002; 93: 1384-1390.

3 Nadel JA, Tierney DF. Effect of a previous deep inspiration on airway resistance in man. J Appl Physiol 1961; 16: 717-719.

4 Salerno FG, Pellegrino R, Trocchio G, et al. Attenuation of induced bronchoconstriction in healthy subjects: effects of breathing depth. J Appl Physiol 2005; 98: 817-821.

5 Salome CM, Thorpe CW, Diba C, et al. Airway re-narrowing following deep inspiration in asthmatic and nonasthmatic subjects. Eur Respir J 2003; 22: 62-68.

6 Scichilone N, Kapsali T, Permutt S, et al. Deep inspiration-induced bronchoprotection is stronger than bronchodilation. Am J Respir Crit Care Med 2000; 162: 910-916.

7 Scichilone N, Bruno A, Marchese R, et al. Association between reduced bronchodilatory effect of deep inspiration and loss of alveolar attachments. Respir Res 2005; 6: 55.
8 Scichilone N, Marchese R, Catalano F, et al. Bronchodilatory effect of deep inspiration is absent in subjects with mild COPD. Chest 2004; 125: 2029-2035.

9 LaPrad AS, West AR, Noble PB, et al. Maintenance of airway caliber in isolated airways by deep inspiration and tidal strains. J Appl Physiol 2008; 105: 479-485.

10 Noble PB, Jones RL, Needi ET, et al. Responsiveness of the human airway in vitro during deep inspiration and tidal oscillation. J Appl Physiol 2011; 110: 1510-1518.

11 Skloot G, Permutt S, Togias A. Airway hyperresponsiveness in asthma: a problem of limited smooth muscle relaxation with inspiration. J Clin Invest 1995; 96: 2393-2403.

12 Chapman DG, King GG, Berend N, et al. Avoiding deep inspirations increases the maximal response to methacholine without altering sensitivity in non-asthmatics. Respir Physiol Neurobiol 2010; 173: 157-163.

13 Crimi E, Saporiti R, Bartolini S, et al. Airway responsiveness to methacholine and deep inhalations in subjects with rhinitis without asthma. J Allergy Clin Immunol 2008; 121: 403-407.

14 Burns GP, Gibson GJ. The apparent response of airway function to deep inspiration depends on the method of assessment. Respir Med 2001; 95: 251-257.

15 Fredberg JJ, Inouye D, Miller B, et al. Airway smooth muscle, tidal stretches, and dynamically determined contractile states. Am J Respir Crit Care Med 1997; 156: 1752-1759.

16 Shen X, Wu MF, Tepper RS, et al. Mechanisms for the mechanical response of airway smooth muscle to length oscillation. J Appl Physiol 1997; 83: 731-738.

17 Wang L, Pare PD, Seow CY. Selected contribution: effect of chronic passive length change on airway smooth muscle length-tension relationship. J Appl Physiol 2001; 90: 734-740.

18 Wang L, Pare PD. Deep inspiration and airway smooth muscle adaptation to length change. Respir Physiol Neurobiol 2003; 137: 169-1678.

19 Noble PB, Sharma A, McFawn PK, et al. Airway narrowing in porcine bronchi with and without lung parenchyma. Eur Respir $J$ 2005; 26: 804-811.

20 Mitchell HW, Sparrow MP. Video-imaging of lumen narrowing; muscle shortening and flow responsiveness in isolated bronchial segments of the pig. Eur Respir J 1994; 7: 1317-1325.

21 Gunst SJ, Stropp JQ, Service J. Mechanical modulation of pressure-volume characteristics of contracted canine airways in vitro. J Appl Physiol 1990; 68: 2223-2229.

22 Noble PB, McFawn PK, Mitchell HW. Responsiveness of the isolated airway during simulated deep inspirations: effect of airway smooth muscle stiffness and strain. J Appl Physiol 2007; 103: 787-795.

23 Croteau JR, Cook CD. Volume-pressure and length-tension measurements in human tracheal and bronchial segments. J Appl Physiol 1961; 16: 170-172.

24 Brusasco V, Pellegrino R, Violante B, et al. Relationship between quasi-static pulmonary hysteresis and maximal airway narrowing in humans. J Appl Physiol 1992; 72: 2075-2080.

25 Noble PB, McFawn PK, Mitchell HW. Intraluminal pressure oscillation enhances subsequent airway contraction in isolated bronchial segments. J Appl Physiol 2004; 96: 1161-1165.

26 Marthan R, Woolcock AJ. Is a myogenic response involved in deep inspiration-induced bronchoconstriction in asthmatics? Am Rev Respir Dis 1989; 140: 1354-1358.

27 Noble PB, West AR, McLaughlin RA, et al. Airway narrowing assessed by anatomical optical coherence tomography in vitro: dynamic airway wall morphology and function. J Appl Physiol 2010; 108: 401-411. 